\title{
Extensive corrosive injuries of the upper airways and gastrointestinal tract
}

\author{
S. Jaillard, MD, ${ }^{a}$ S. Nseir, MD, ${ }^{b}$ D. Métois, MD, ${ }^{a}$ C. H. Marquette, MD, ${ }^{c}$ J. Darras, MD, ${ }^{d}$ H. Porte, MD, a and \\ A. Wurtz, MD, ${ }^{a}$ Lille, France
}

1 26-year-old man was referred to our intensive care unit shortly after having ingested a massive amount of a caustic agent (200 mL of potassium hydroxide) with suicidal intent. He had epigastric tenderness, severe oropharyngeal burns, and respiratory distress. In view of the severity of the injury, esophageal endoscopy could not be performed. Examination with a fiberoptic bronchoscope revealed extensive necrosis of the posterior wall of the tracheobronchial tree from the proximal trachea to the distal ends of both main bronchi. The patient was started on a regimen of intravenous broad-spectrum antibiotics (piperacillin/tazobactam and amikacin). A decision was made to perform emergency surgery, which began 9 hours after the ingestion.

Exploratory laparotomy was performed initially. Severe injuries to the gastrointestinal tract necessitated extensive resection of necrotic organs. A total gastrectomy, cephalic duodenopancreatectomy extending to $30 \mathrm{~cm}$ of the jejunum, and a cholecystectomy were performed. Biliodigestive and pancreaticodigestive anastomoses were created. A feeding jejunostomy tube was also placed. Cervical esophagostomy was then performed over a large rubber tube. The patient was reintubated with a long left-sided endobronchial tube over a flexible bronchoscope. A right thoracotomy was performed and severe mediastinitis was evident. Necrosis involved all layers of the esophagus including the periesophageal tissues. Gentle dissection was used to remove the esophagus, with care taken to preserve the tracheobronchial tree. After pleural and mediastinal irrigation, the posterior tracheal membrane appeared grayish, but without visible perforation. So that further deterioration and complications could be avoided, the posterior aspects of the trachea and main bronchi were patched with the right lung (Figure 1, A).

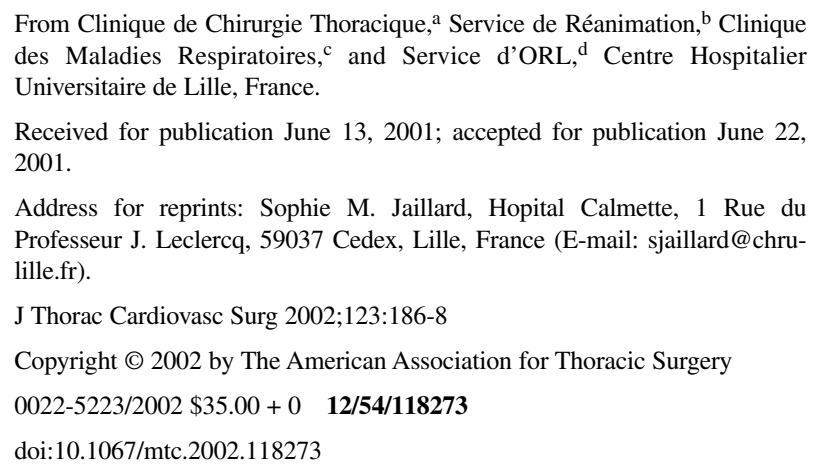

Postoperatively, massive necrosis of laryngeal and pharyngeal wall mandated immediate tracheostomy. Subsequent intense medical and surgical management was required (Figure 1, B). A fiberoptic bronchoscope was used daily to assess the evolution of airway necrosis and to clear bronchial secretions and necrotic tissues. On the third postoperative day, a fissure at the level of the main carina was noted. Progressive necrosis involved the entire posterior wall of the trachea and main bronchi. The patient continued to receive mechanical ventilation for 10 days with low peak inspiratory pressures $\left(<20 \mathrm{~cm} \mathrm{H}_{2} \mathrm{O}\right)$ before being weaned from the ventilator. A large mediastinal cavity developed contiguous to the carinal fissure. Repeated daily bronchoscopic tracheal suctioning was combined with mediastinal drainage. Progressive improvement was seen with resolution of the air leak, and definitive bronchial cicatrization was observed at 12 weeks (Figure 2). Concurrently, gastrointestinal recovery was uneventful, and the tracheostomy was kept in place because of the extensive pharyngeal and laryngeal destruction.

At 7 months, a gastrointestinal evaluation disclosed that colonic transposition for esophageal replacement would be feasible, despite the patient's history. At that time, the patient had to choose between deglutition and phonation because of complete destruction of the larynx. Reintervention was planned in the setting of a favorable response to psychiatric treatments. Reconstruction was performed by retrosternal left colon interposition. The destroyed pharyngolarynx was resected. The proximal colonic anastomosis was tailored onto the pharynx and the distal anastomosis onto the jejunum. The postoperative course was uneventful other than some initial difficulty with swallowing. One year later, the patient can eat normally and keeps the tracheostomy cannula in place at night to maintain the stoma.

\section{Discussion}

Both tracheobronchial injuries and duodenopancreatic injuries remain poor prognostic factors after lye ingestion. ${ }^{1-3}$ We therefore report the successful treatment of extensive burns related to massive ingestion of a strong caustic agent. Considering the severity of the abdominal and thoracic lesions, healing was remarkable and, in our opinion, the result of the short delay between ingestion and extensive optimal surgical treatment, as well as the intense postoperative care given to a young patient without underlying health problems.

In our case, early bronchoscopic examination revealed tracheobronchial involvement caused by necrotic extension from the burned esophagus. There was no evidence of direct aspiration of the caustic agent, and lesions affected only the posterior wall of the trachea and of both main bronchi. Early blunt esophagectomy, while usually indicated in transmural necrosis of the esophagus, ${ }^{4}$ 

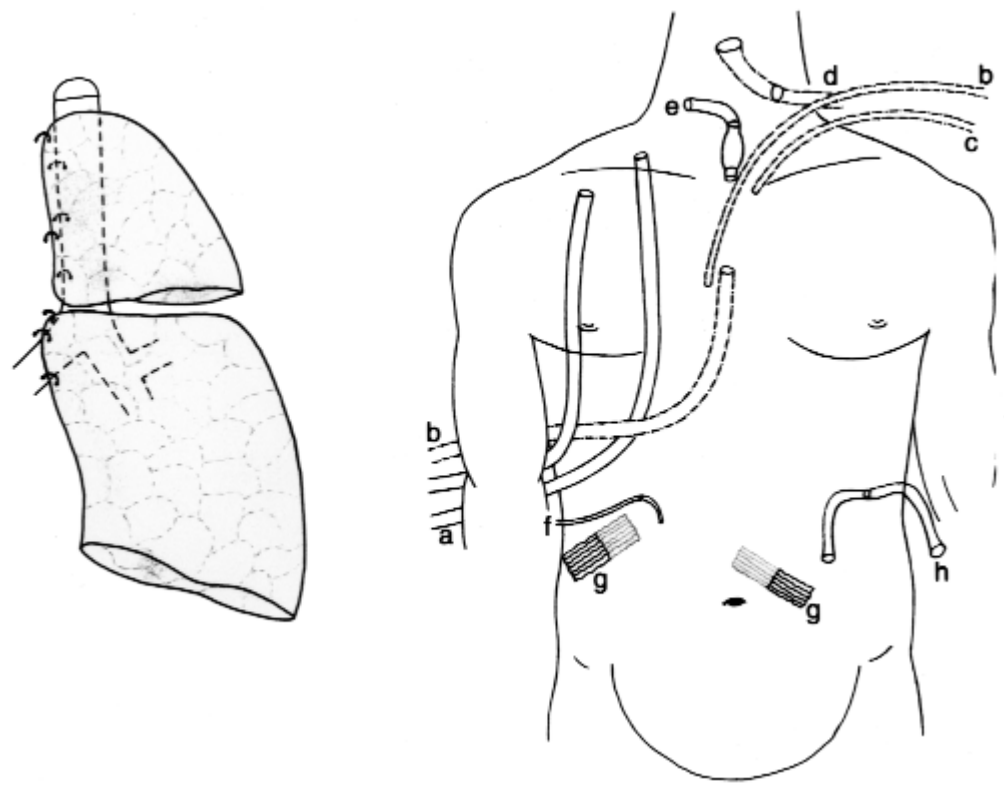

Figure 1. Surgical management: A, Pulmonary patch: both the upper and the lower lobes were mobilized and secured onto the remaining intact mediastinal structures (periaortic tissue and cartilage-membranous angle of the airway). B, Schematic postoperative view. $a$, Pleural tube; $b$, mediastinal tube (one drain inserted via a cervical approach and the second via the inferior chest wall); $c$, cervical tube; $d$, esophagostomy; $e$, tracheostomy; $f$, biliary tube; $g$, anastomotic rubber foil; $h$, feeding jejunostomy.
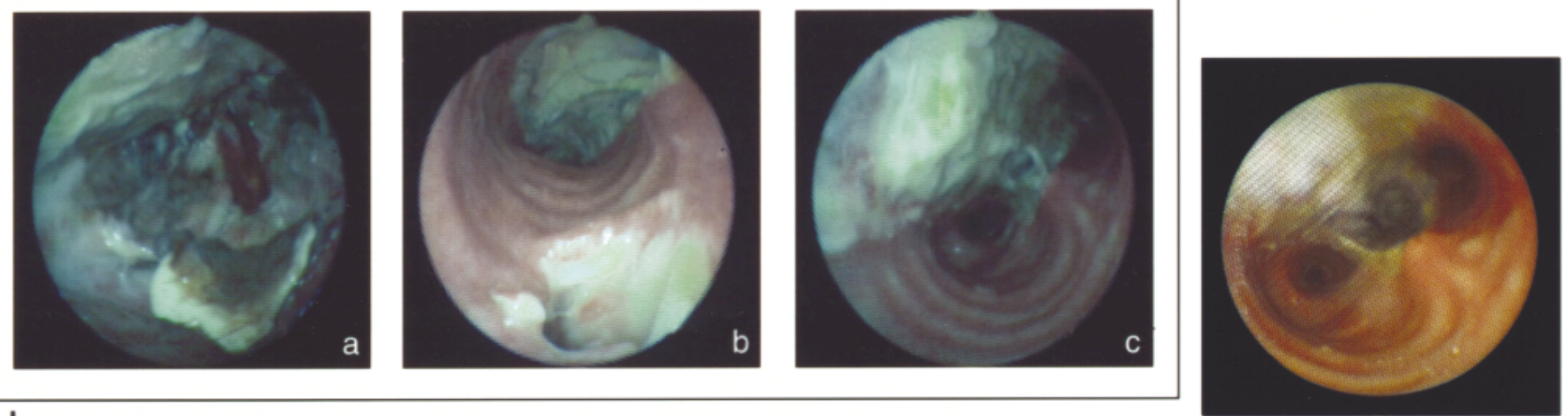

I

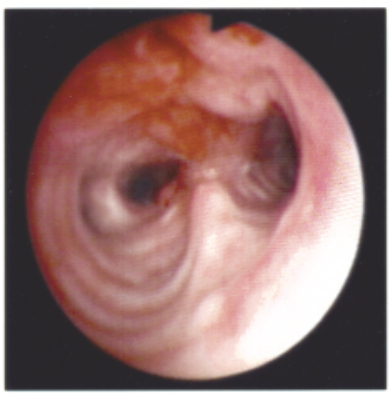

III
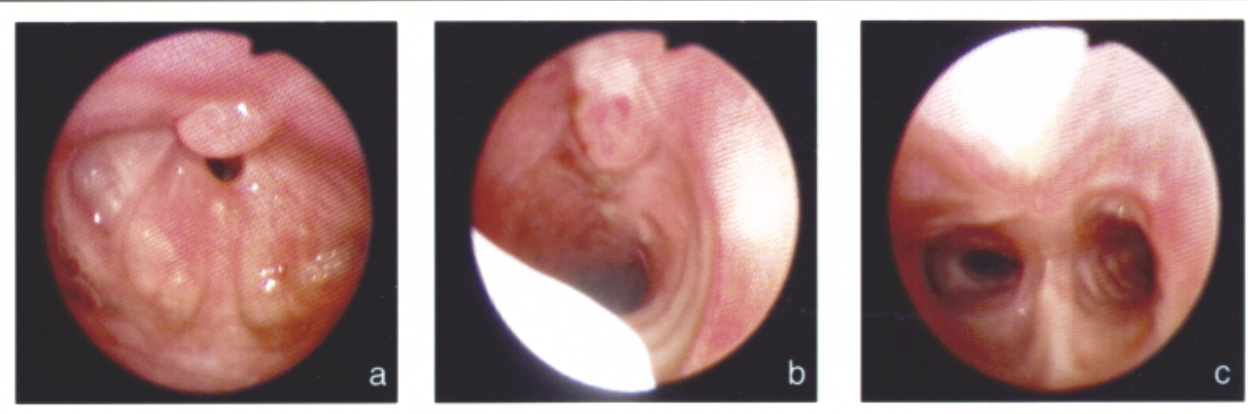

IV

Figure 2. Endoscopic follow-up. I, Early lesions at day 5: a, oropharyngeal necrosis; b, tracheal necrosis, with superior mediastinal tube visualized in front of the tracheostoma; $c$, main carina destruction. II, Lesions at day 15. III, Lesions at 2 months. IV, Sequelae: a, complete destruction and stenosis of the oropharynx; $b$, complete tracheal healing facing the cannula; $c$, aspect of the main carina. 
was contraindicated and dangerous in this case because of the risk of worsening intraoperative and postoperative air leakage. It was therefore necessary to operate through a right thoracotomy. So that the possibility of air leak during esophagectomy could be avoided, a long left bronchial tube was positioned distally in the left main bronchus. Artificial ventilation by high-frequency jet ventilation would have been another option.

This case clearly demonstrates the usefulness of a pulmonary patch (as described by Sarfati and associates ${ }^{1}$ ) to prevent air leakage and the potential for "spontaneous" healing of tracheobronchial perforations.

\section{References}

1. Sarfati E, Jacob L, Servant JM, d'Acremont B, Roland E, Ghidalia T, et al. Tracheobronchial necrosis after caustic ingestion. $J$ Thorac Cardiovasc Surg. 1992;103:412-3.

2. Cattan P, Munoz-Bongrand N, Berney T, Halimi B, Sarfati E, Celerier M. Extensive abdominal surgery after caustic ingestion. Ann Surg. 2000;231:519-23.

3. Wu MH, Lai WW, Hwang T, Lee SC, Hsu HK, Lin TS. Surgical results of corrosive injuries involving esophagus to jejunum. Hepatogastroenterology. 1996;43:846-50.

4. Gossot D, Sarfati E, Celerier M. Early blunt esophagectomy in severe caustic burns of the upper digestive tract: report of 29 cases. $J$ Thorac Cardiovasc Surg. 1987;94:188-91. 